\title{
The Impact of the Indian X-ray satellite, AstroSat, on Accretion Processes in Cosmic Sources
}

\section{Kulinder Pal Singh ${ }^{* \dagger}$}

Tata Institute of Fundamental Research, Homi Bhabha Road, Mumbai 4000005, India

E-mail: singhetifr.res.in

\begin{abstract}
AstroSat is the first space astronomy observatory launched by India. It was put into a near equatorial (inclination of $6^{\circ}$ ) orbit $650 \mathrm{~km}$ above the Earth on 28 September 2015, by the Indian Space research Organisation. AstroSat carries many telescopes and detectors to observe cosmic sources over a wide wavelength band, from optical/UV to hard X-rays. All the payloads onboard the satellite have been operating successfully since launch. Several observations have been carried out and the performance verification and calibration have been completed. The Observatory is now in full science operation, and is open to the Indian scientific community since October 2016 for observations. A very brief introduction to the capabilities of the payloads and some of the early observational results are presented here.
\end{abstract}

Accretion Processes in Cosmic Sources, APCS2016

5-10 September 2016,

Saint Petersburg, Russia

* Speaker.

${ }^{\dagger}$ A footnote may follow. 


\section{AstroSat and its Scientific Instruments}

AstroSat is India's first multi wavelength observatory. Weighing about 1.5 tons, it was launched into a circular orbit $650 \mathrm{kms}$ above the Earth on September 28, 2015 from India's spaceport in Shriharikota, near Chennai in India by the C-30 Polar Satellite Launch Vehicle. AstroSat carries a suite of co-aligned Optical, UV and X-ray telescopes and detectors to observe the Universe. In particular, AstroSat carries the following main scientific payloads (see Figure 1): (i) an Ultra-Violet Imaging Telescope (UVIT) having two independent telescopes Đ one for far-UV (FUV) and another for near-UV (NUV) and visible wavelengths, (ii) a Soft X-ray Telescope (SXT), (iii) three Large Area Xenon Proportional Counters (LAXPCs), (iv) a Cadmium-Zinc-Telluride Imager (CZTI), and (v) a Scanning Sky Monitor (SSM). Three co-aligned X-ray instruments onboard with overlapping energy response provide a very wide-band energy range. The co-aligned UV and optical telescopes provide simultaneous optical, UV and X-ray observations of any cosmic object in the sky, making it a unique mission in X-ray and UV astronomy. The orbital inclination of $6^{\circ}$ provides a low particle background for the detectors aboard the AstroSat.

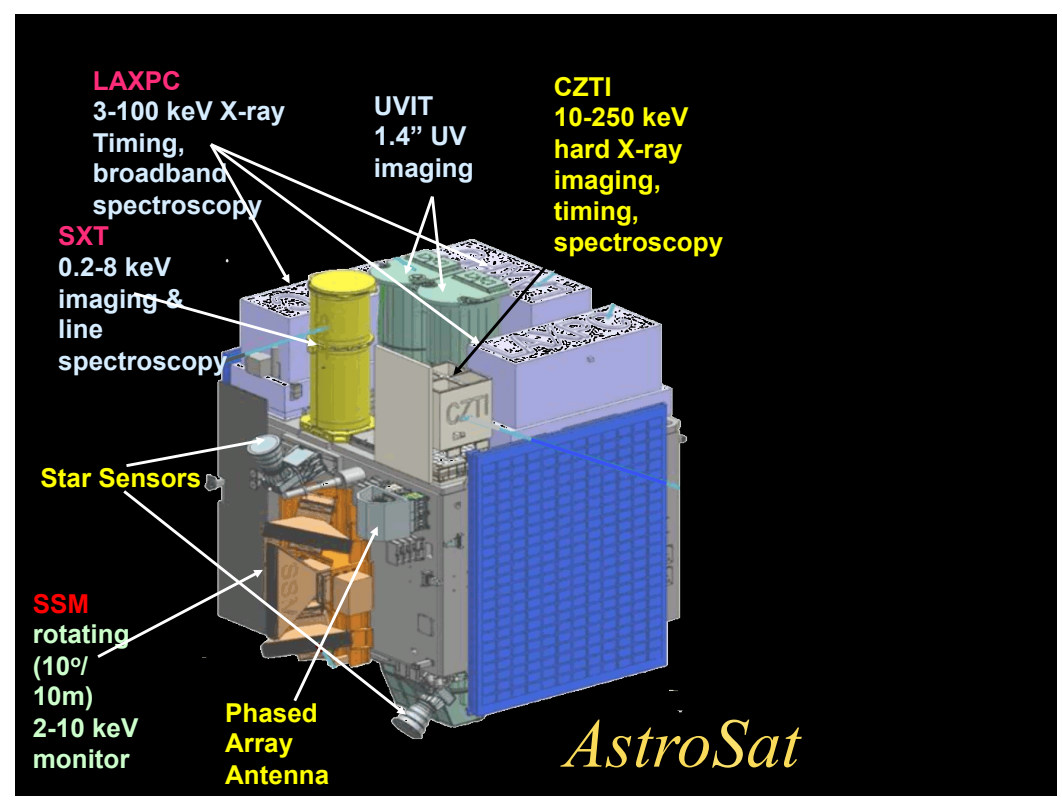

Figure 1: A schematic of the AstroSat with folded solar panels showing its principal instruments.

The main characteristics of the telescopes and detectors aboard the AstroSat are summarised below: (a) UVIT has 2 Telescopes one for the FUV and another one configured for the NUV and Vis and with total bandwidth of $1300-5500$ Angstroms. Its angular resolution is $1.4 \operatorname{arcsec}$ (FUV, NUV); and 2.2 arcsec (Vis), with the field of View of 28 arcmin (Dia.). It carries a total of 13 filters and gratings and has time resolution of $1.7 \mathrm{~ms}$. The effective areas with different filters in the FUV, NUV and the visible channels are displayed in Figures 2, 3 and 4 respectively. The angular resolution of $\sim 1.4 \operatorname{arcsec}$ is $\sim 3$ times better than that of the Galex.

(b) The LAXPC has 3 units named as LX10, LX20, LX30 each having a bandwidth of 3-80 $\mathrm{keV}$ with max. effective area of $8000 \mathrm{~cm}^{2}$ in $3-20 \mathrm{keV}$ for all 3 units combined. A picture of one of the LAXPC units is shown in Figure 5. The sensing gas is mostly Xenon at 2 atmospheres 


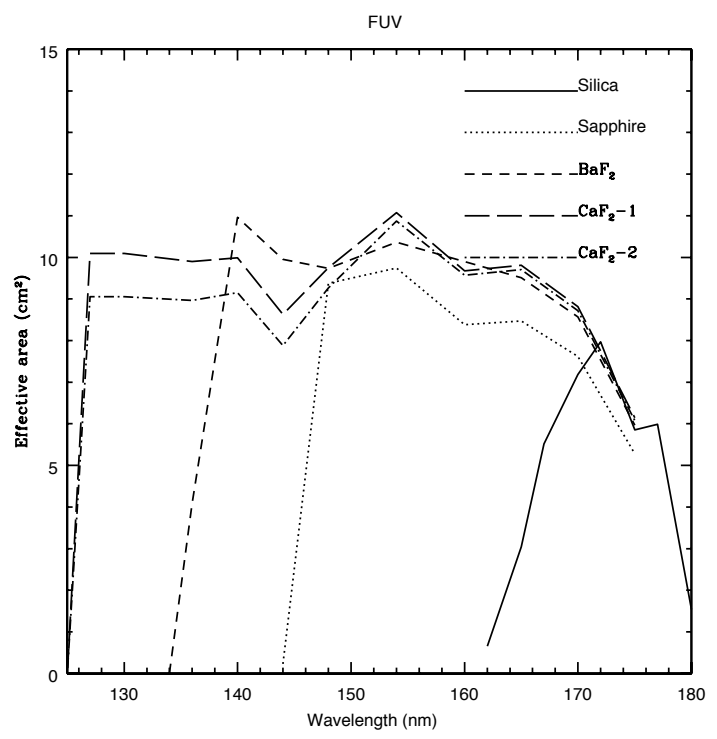

Figure 2: Transmission curves for the different filters in the FUV channel of the UVIT.

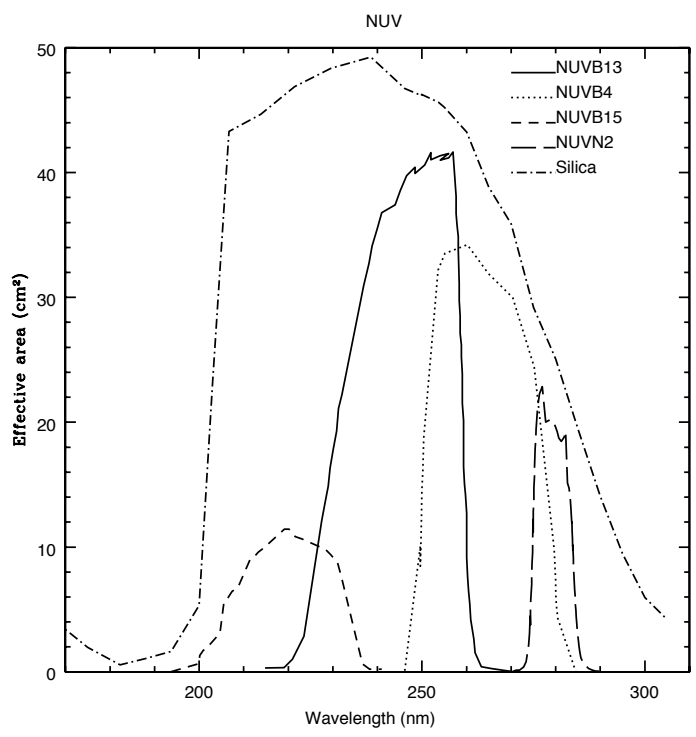

Figure 3: Transmission curves for the different filters in the NUV channel of the UVIT.

providing a significant area between $20-80 \mathrm{keV}$ which provides a significant advantage over the $R X T E$. The field of view is defined by mechanical collimator and is $1^{\circ} \times 1^{\circ}$. The energy resolution is $12 \%$ at $22 \mathrm{keV}$, and the time resolution is $10 \mu \mathrm{s}$.

(c) The CZTI is a coded mask imager with Cd-Zn-T detector cooled passively by a cold plate. It has a bandwidth of $10-100 \mathrm{keV}$ and an angular resolution of 8 arcmin. Its maximum effective Area is $1000 \mathrm{~cm}^{2}$, and its field of view is $6^{\circ} \times 6^{\circ}$. The energy resolution is $5 \%$ at $100 \mathrm{keV}$, and the time Resolution is $1 \mathrm{~ms}$. The CZTI has the capability to measure the polarisation signals above $100 \mathrm{keV}$ (Chattopadhyay, et al. 2014). 


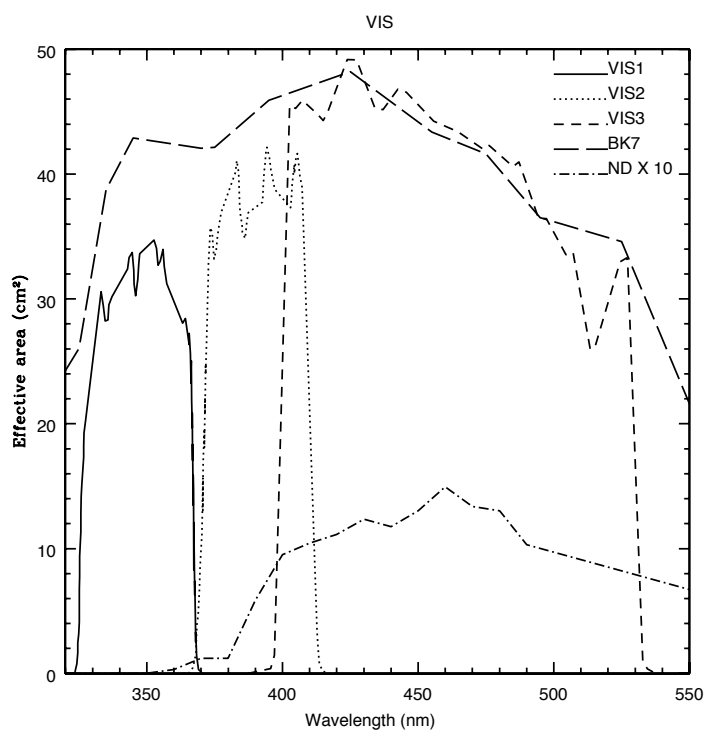

Figure 4: Transmission curves for the different filters in the Visible channel of the UVIT.

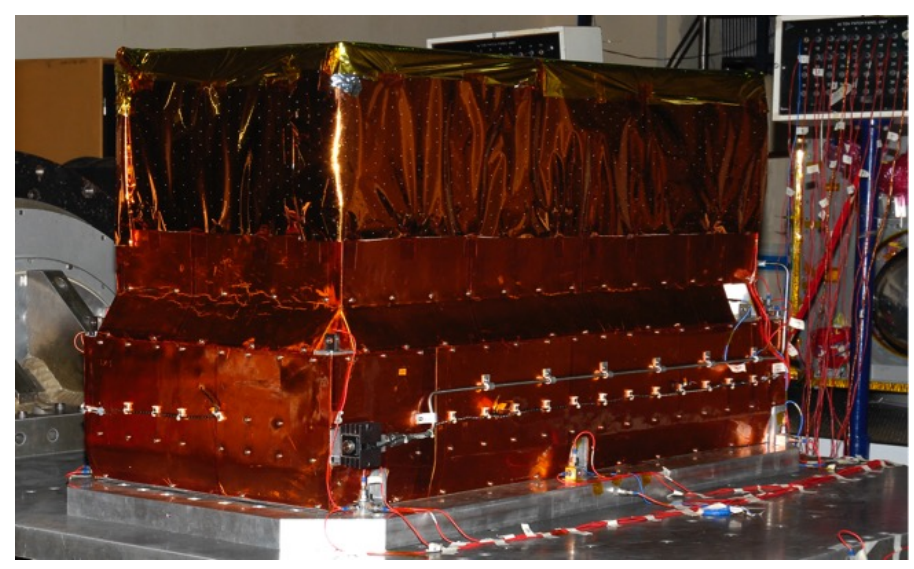

Figure 5: One of the units of the LAXPC

(d) The SXT is grazing incidence telescope with 2 metres focal length with a cooled MOS $\mathrm{CCD}\left(\right.$ at $-80^{\circ} \mathrm{C}$ ) in the focal plane. It has 40 co-axial shells of mirrors with gold surfaces replicated from smooth glass surfaces coated with sputtered gold. The mirrors are assembled quadrant wise in approximate Wolter-I geompetry with maximum apertue of $260 \mathrm{~mm}$ and minimum aperture of 130 $\mathrm{mm}$. SXT has a bandwidth of $0.2-8.0 \mathrm{keV}$ with maximum effective area of $\sim 90 \mathrm{~cm}^{2}$ at $1.5 \mathrm{keV}$. The angular resolution is $2 \operatorname{arcmin}$ (FWHM) and $10 \mathrm{arcmin}$ (HPD). The field of view is $40 \mathrm{arcmin}$ (dia) and the plate scale in the focal plane is 4.0 arcsec per pixel. The energy resolution is $\sim 140$ $\mathrm{eV}$ at $6 \mathrm{keV}$. The time Resolution is 2.37s in the default PC (Photon Counting) mode and 0.278s in the FW (Fast Window) mode. The SXT has a very good energy resolution and low background and can detect sources down to $20 \mu \mathrm{Crab}$ level.

(e) The SSM consists of 3 Units mounted on the side and rotating to cover the sky. with an angular resolution of $12 \operatorname{arcmin} \times 2.5^{\circ}$. The energy bandwidth is $25-10 \mathrm{keV}$ and the maximum 


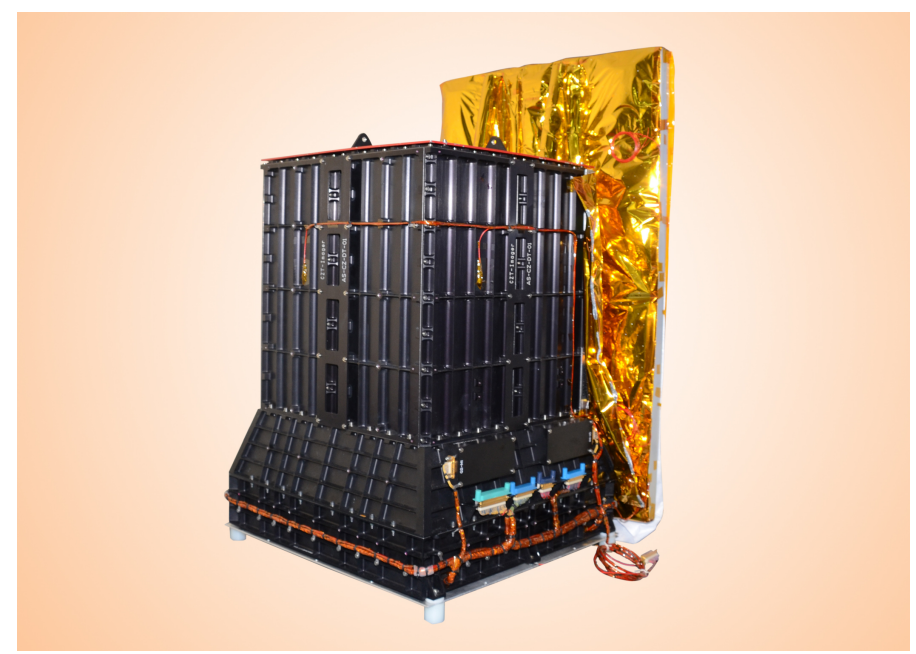

Figure 6: The CZTI detector with its cold plate assembly.

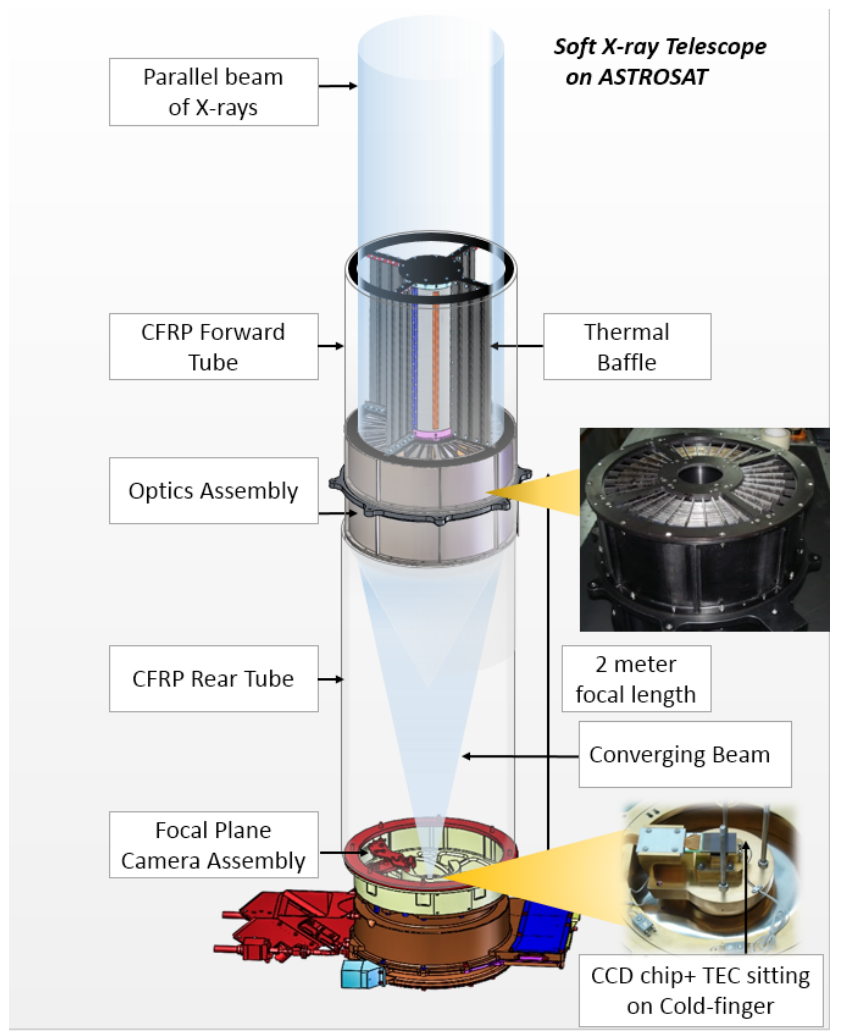

Figure 7: A schematic of the SXT showing its various parts. 
effective area is $53 \mathrm{~cm}^{2}$ at $5 \mathrm{keV}$ and the field of view is $22^{\circ} \times 100^{\circ}$ for the central unit and $26.8^{\circ}$ $\times 100^{\circ}$ for the edge units. The energy resolution is $25 \%$ at $6 \mathrm{keV}$, and the time resolution is $1 \mathrm{~ms}$.

A more detailed description of the AstroSat is given in Singh et al. (2014), and that of its principal instruments in (Bhalerao et al. 2017, Ramadevi et al. 2017, Singh, et al. 2016, 2017a, Subramaniam, et al. 2016, Tandon et al. 2017a, Vadawale et al. 2016, Yadav et al. 2016a).

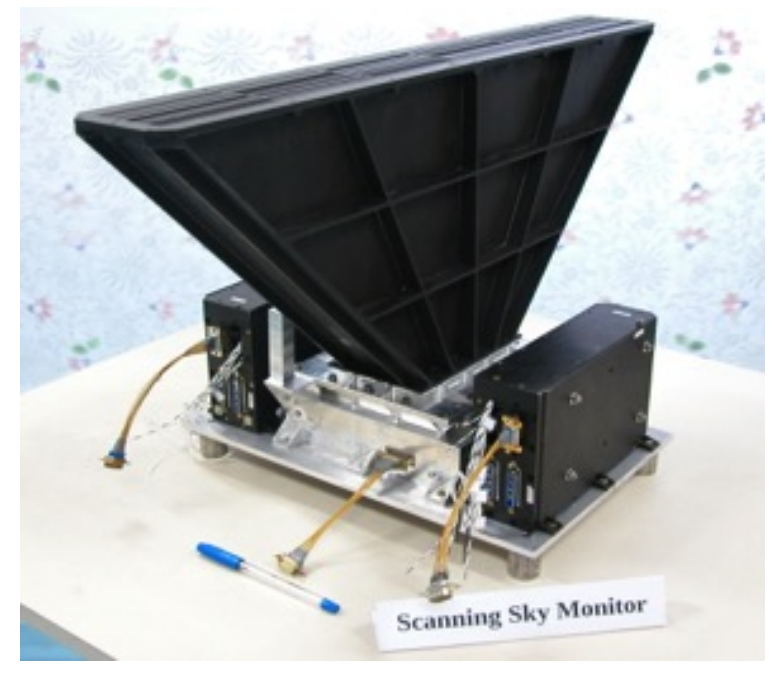

Figure 8: One of the units of the SSM.

\section{Observations with the AstroSat}

AstroSat provides a very wide X-ray band combined with UV and optical telescopes for simultaneous observations of variable sources in the sky. Several observations have been carried out in the last one year with a large number of them directed towards its calibration. Nevertheless several interesting new results have been obtained. UV imaging of normal, starburst and dwarf galaxies is addressing the evolution of stellar populations in them. NUV and FUV images of a galaxy, NGC 2336, are shown in Figures 9 and 10.

A hot star (T 17000 $\pm 500 \mathrm{~K}$ ), suspected to be a post-AGB/HB (Asymptotic Giant Branch/ Horizontal Branch) star and companion to a cool star (T 6000 $\pm 150 \mathrm{~K})$ known as a Blue Straggler Star (BSS) has been discovered through UV imaging of the open cluster NGC 188 by Subramaniam et al. (2017). RR Lyrae variable stars have been detected using time lapse images of the globular cluster NGC 1851. Other examples of UVIT results are multiple rings of star formation in NGC 7217, images of the Small Magellanic Cloud, time variability during a recent re-brightening of an Intermediate Polar, FO Aqr, etc. (see Tandon et al. 2017b).

Several $\gamma$-ray bursts have been detected with the CZTI, and one such burst is shown in Figure 11. A polarisation signal has been measured at $2.5 \sigma$ level for a Gamma Ray Burst (GRB) of fluence less than $2 \times 10^{-5} \mathrm{ergs} \mathrm{cm}^{-2}$. More significant detections are expected to become possible for brighter GRBs are expected and that should help to clearly distinguish between various models of GRB prompt emission mechanism (Rao et al. 2016). The sensitivity of the CZTI is quite close to that of the Fermi GBM and therefore it should be able to detect possible hard X-ray emission associated with gravitational wave events. 


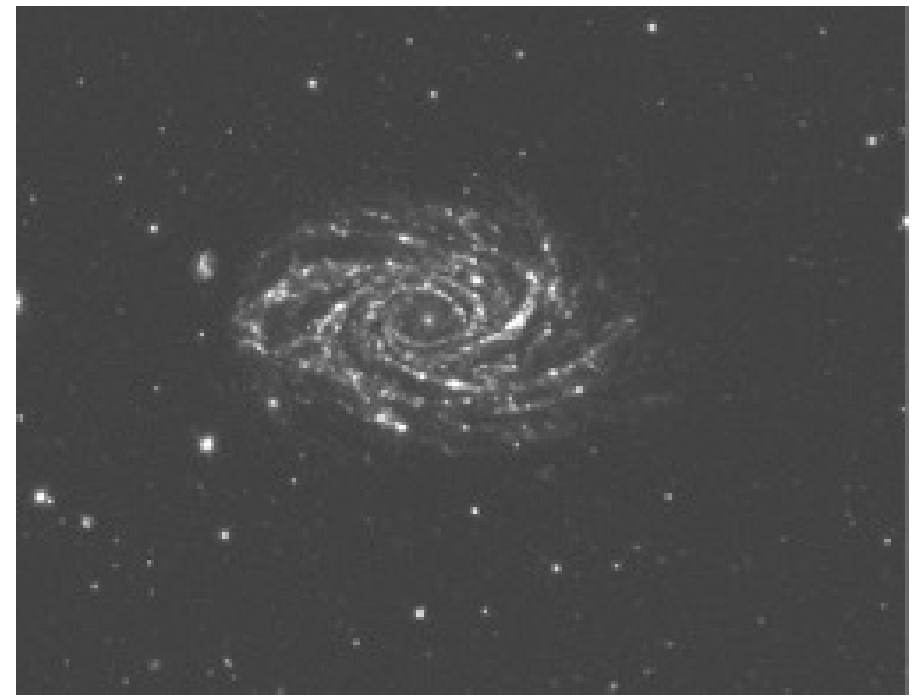

Figure 9: Image of the NGC2336 taken with UVIT in the NUV. Credits: S.N. Tandon+UVIT team

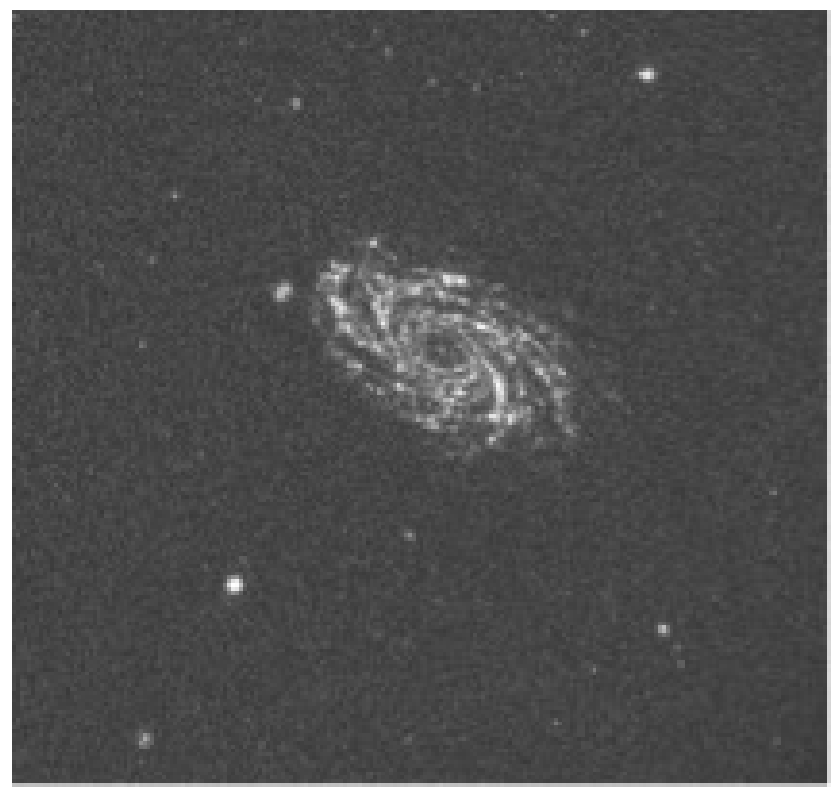

Figure 10: Image of the NGC2336 taken with UVIT in the FUV. Credits: S.N. Tandon+UVIT team

Several Galactic black-hole and neutron star binaries have been observed with the LAXPC detectors. X-ray light curves as observed with the LAXPC units of one high mass neutron star binary, 4U0115+63, are shown in Figure 12. Observations with LAXPC caught the micro quasar GRS1915+105 in a rare $\chi$ mode revealing many new features in their energy dependent power spectra, e.g., strong low-frequency $(2.55,4.53,6.55 \mathrm{~Hz})$ quasi-periodic oscillations (QPOs) and their harmonics along with broadband noise, and with the QPO frequency changing rapidly (in a few hours) with source intensity (Yadav et al. 2016). In Cyg X-1 the power spectrum obtained by LAXPC shows two broad Lorentzian functions centered at 0.4 and $3 \mathrm{~Hz}$ with the rms of the low-frequency component showing a decrease from $15 \%$ at $4 \mathrm{keV}$ to $10 \%$ at $50 \mathrm{keV}$, while the 


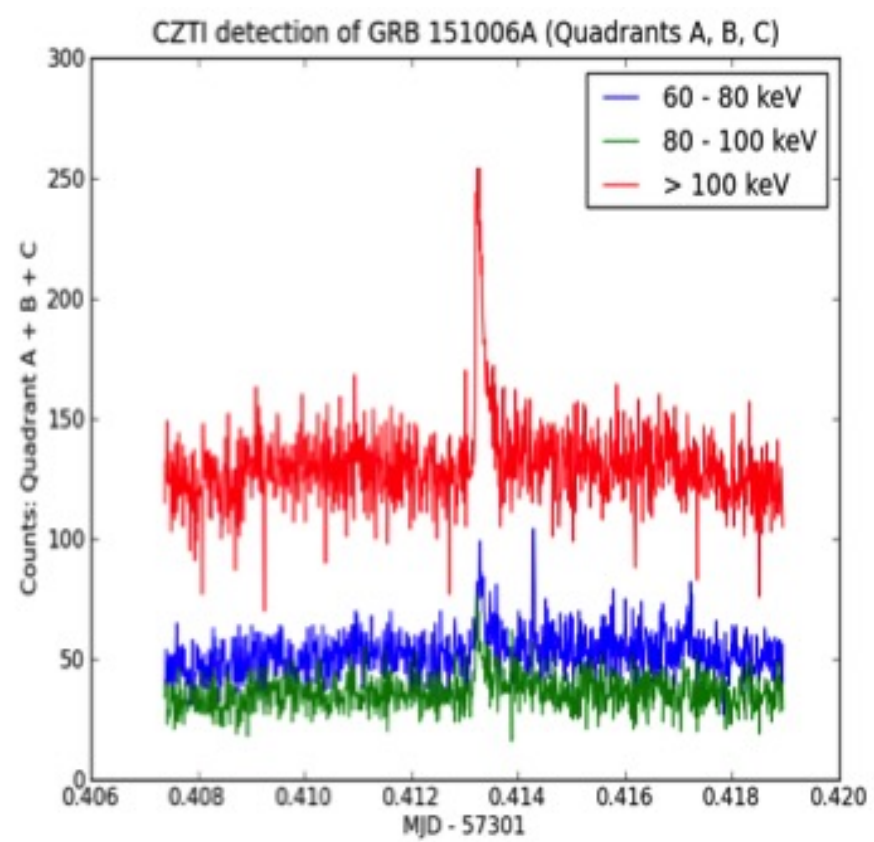

Figure 11: A $\gamma$-ray burst detected with the CZTI. Credits: A.R. Rao+CZTI team

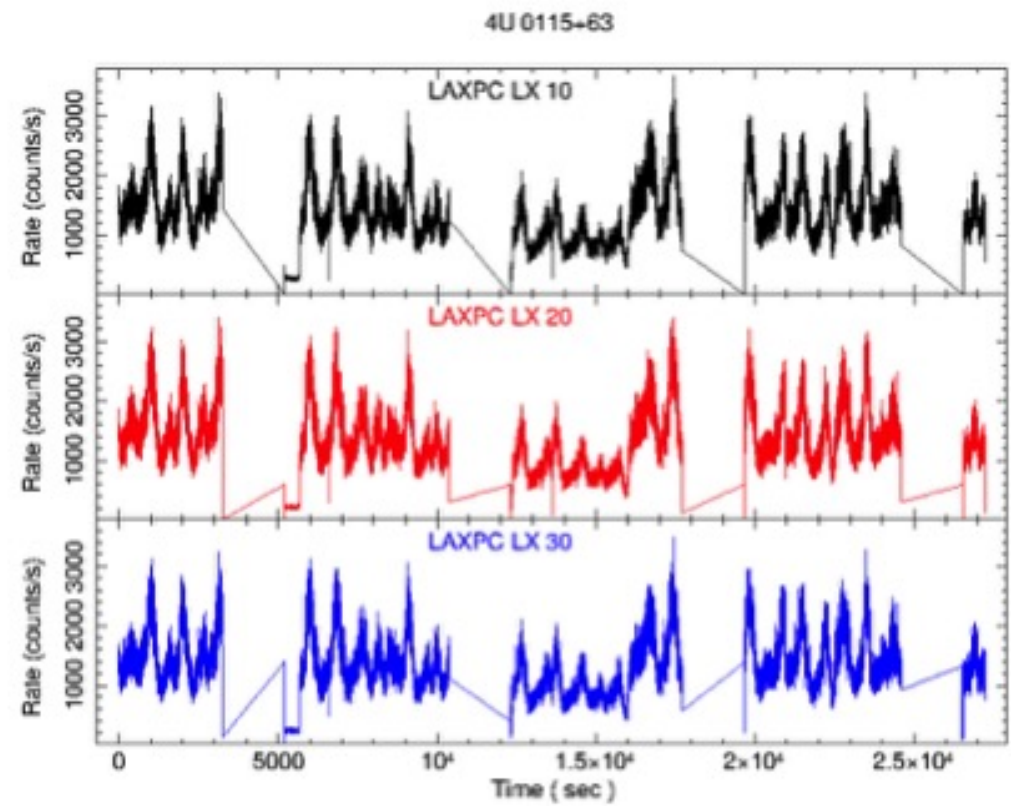

Figure 12: X-ray pulsations from $4 \mathrm{U} 0115+63$ in the three LAXPC detectors. Credits: J.S. Yadav+LAXPC team 
time lag between the hard (20-40 keV) and soft (5-10 keV) bands increase with energy for both the low and high-frequency components (Misra et al. 2017). Such observations are helping us to understand the dynamics of X-ray binaries with black-hole companions.
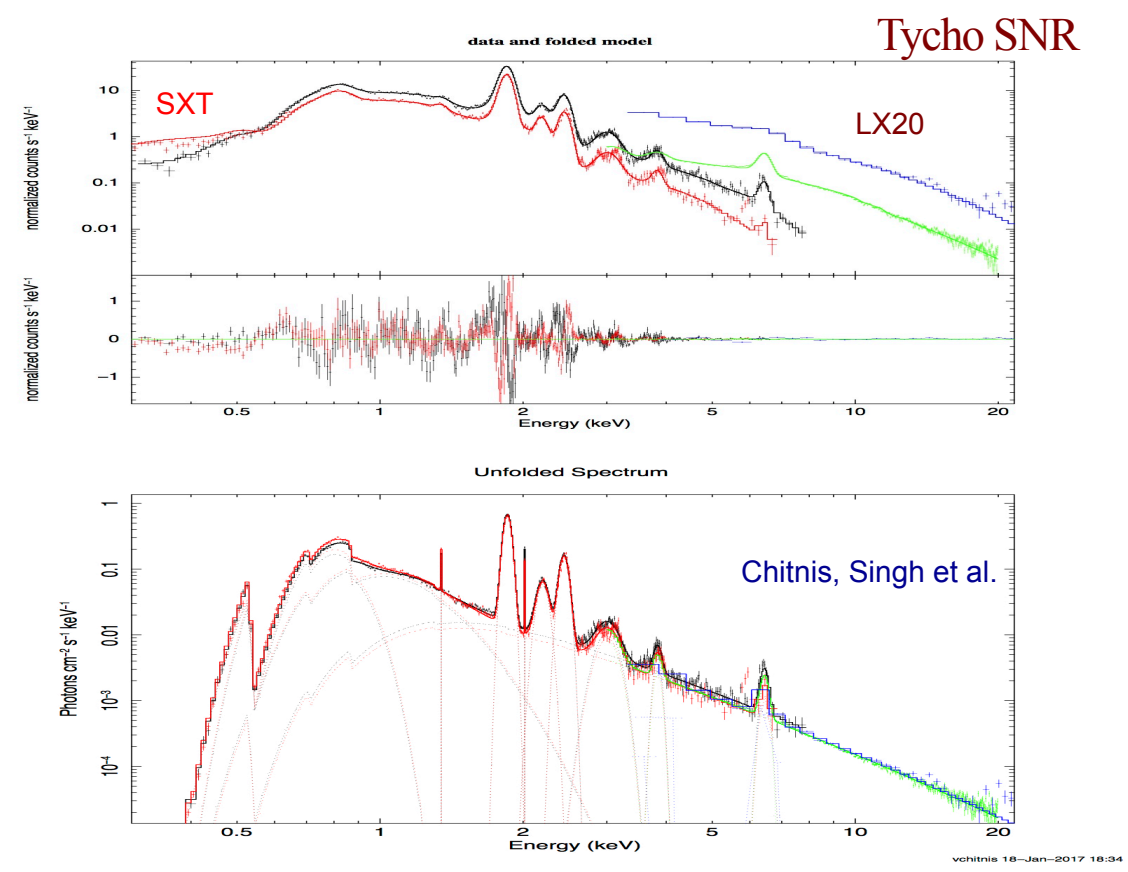

Figure 13: X-ray spectrum of Tycho supernova remnant with the SXT (red), Swift-XRT (black), NuS$\operatorname{tar}($ green) and the LAXPC unit 2(blue). Credits:SXT team

X-ray spectra and light curves using the SXT have been taken of many kinds of sources- stellar coronae, magnetic Cataclysmic Variables (mCVs), supernova remnants, X-ray binaries, AGN, clusters of galaxies. Figure 13 shows the SXT spectrum of Tycho supernova remnant and its comparison with the spectrum taken with the Swift-XRT, NuStar and LAXPC. Our SXT observations of Cyg X-3, an enigmatic X-ray binary, when used in conjunction historical data of 45 years has led to a new binary ephemeris for the 4.8 hour period and an improved period derivative. We do not find any evidence for a second derivative in the period variation of Cyg X-3 (Bhargava et al. 2017) Observations with the SXT are providing variability on long time scales of minutes to days in many active galactic nuclei (AGN) (Singh et al. 2017b), and complementing the LAXPC spectral data with simultaneously obtained high quality spectra. Wide-band X-ray spectroscopy has been carried out for Crab, Cyg X-1, several blazars and Seyferts, and a few mCVs using the simultaneous observations with the SXT and the LAXPC (Singh \& Bhattacharya 2017). Our wide-band SXT+LAXPC spectra of an Integral discovered mCV, IGR J15094-6649, while confirming the spin period of $808.4 \mathrm{~s}$ show that the bremsstrahlung temperature (kT) is $29 \mathrm{keV}$ and not $14 \mathrm{keV}$ as was previously measured with the RXTE (Singh et al. in preparation).

The AstroSat was specially oriented to observe some binary X-ray sources with the SSM so as to check its performance and calibration. Figure 14 shows the X-ray intensity variations from the black-hole binary GRS1915+105. 


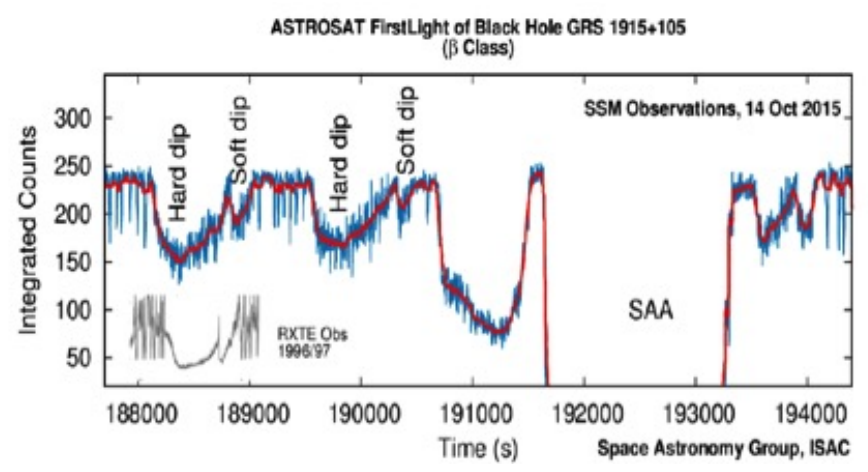

Figure 14: X-ray light curve of GRS1915+105 as observed with the SSM detectors. Credits: Ramadevi+SSM team

\section{References}

[1] T. Chattopadhyay, S. V. Vadawale, A. R. Rao, S. Sreekumar, \& D. Bhattacharya, ExA, 37, 555 (2014)

[2] K. P. Singh, et al., ASTROSAT Mission, Proc. of SPIE, Space Telescopes and Instrumentation 2014: UV to Gamma Ray, 9144 [doi : $10.1117 / 12.2062667$ ].

[3] V. Bhalerao, D. Bhattacharya, A. Vibhute, et al. JoAA, 2017, in press

[4] M. C. Ramadevi, S. Seetha, D. Bhattacharya, et al., JoAA, 2017, 38, 32.

[5] Singh, K. P., Stewart, G. C., Chandra, S., et al., Proc. of SPIE, Space Telescopes and Instrumentation 2016: Ultraviolet to Gamma Ray, Eds: Jan-Willem A. den Herder, Tadayuki Takahashi, Marshall Bautz 9905, (99051E), [doi : $10.1117 / 12.2235309$ ]

[6] K. P. Singh, G. C. Stewart, N. J. Westergaard, et al. JoAA, 2017a, 38, 29.

[7] A. Subramaniam, S. N. Tandon, J. Hutchings, et al. Proc. of SPIE, Space Telescopes and Instrumentation 2016: Ultraviolet to Gamma Ray, Eds: Jan-Willem A. den Herder, Tadayuki Takahashi, Marshall Bautz, 9905. (99051F). [doi: $10.1117 / 12$.2235271] (arxiv: 1608.01073)

[8] S. N. Tandon, J. B. Hutchings, S. K. Ghosh, et al. JoAA, 2017a, 38, 28

[9] S. V. Vadawale, A. R. Rao, D. Bhattacharya, et al. Proc. of SPIE, Space Telescopes and Instrumentation 2016: Ultraviolet to Gamma Ray, Eds: Jan-Willem A. den Herder, Tadayuki Takahashi, Marshall Bautz, 9905, (99051F)

[10] J. S. Yadav, P. C. Agrawal, H. M. Antia, et al. Proc. of SPIE, Space Telescopes and Instrumentation 2016a: Ultraviolet to Gamma Ray, Eds: Jan-Willem A. den Herder, Tadayuki Takahashi, Marshall Bautz, 9905, (99051D), [doi : $10.1117 / 12$.2231857]; and ApJ, 2016, 833, 27

[11] A. Subramaniam, N. Sindhu, S. N. Tandon, et al. ApJL, 2017, 833, L27

[12] S. N. Tandon, S. K. Ghosh, J. Hutchings, S. Stalin, A. Subramaniam, Current Science (submitted), 2017 b.

[13] A. R. Rao, V. Chand, M. Hingar, et al, ApJ, 2016, 833, 86

[14] Y. Bhargava, A. R. Rao, K. P. Singh et al., ApJ (submitted), 2017.

[15] K. P. Singh and D. Bhattacharya, Current Science (submitted), 2017. 